\title{
Conditional Density Matrix in the Context of Noncontextuality
}

\author{
V.Belokurov, O.Khrustalev, V.Sadovnichy and O.Timofeevskaya \\ Moscow State University, 119992, Moscow, Russia \\ e-mail: olga@goa.bog.msu.ru
}

\begin{abstract}
Conditional density matrix represents a quantum state of subsystem in different schemes of quantum communication. Here we discuss some properties of conditional density matrix and its place in general scheme of quantum mechanics.
\end{abstract}

Talk presented at the Eleventh Lmonosov Conference on Elementary Particle Physics, Moscow, August, 2003.

\section{Introduction}

A problem of a correct quantum mechanical description of divisions of quantum systems into subsystems and reunification of subsystems into new joint systems attracts a great interest due to the present development of quantum communication.

In principle the theory of such processes was created in general by von Neumann in 1927 [1] when he constructed deductive scheme of quantum mechanics.

Nevertheless until now the description of similar processes and their interpretation involves some problems. For example, a recent entanglement swapping experiment raised discussion. The authors [2] finished their interpretation of experimental results about entanglement swapping and teleportation by quotation: this paradoxes do not arise if the correctness of quantum mechanics is firmly believed.(A.Peres). This statement does little to explain why there is no paradox and it is difficult to agree with it because quantum mechanics is a science.

Quantum mechanics predicts probabilities for various possible outcomes of measurement once we specify the procedure used for preparation of the physical system. In quantum mechanics the important assumption, which might be called the "noncontextuality" of probabilities, means that probabilities are consistent with the Hilbert-space structure of the 
observable. With these assumptions the probabilities for all measurements can be derived from a density operator $\hat{\rho}$ associated by von Neumann with quantum mechanical state.

The quantum states of subsystem of complicated system are described by reduced and conditional density matrices. This approach helps to avoid difficulties and paradoxes in interpretation of some delicate experiments in different schemes of quantum communication.

\section{The State in Quantum Mechanics}

\subsection{Kinematic Quantum Mechanics Postulate}

In quantum mechanics

each dynamical variable $\mathcal{F}$ of a system $\mathcal{S}$ corresponds to a linear operator $\hat{F}$ in Hilbert space $\mathcal{H}$

$$
\text { dynamical variable } \mathcal{F} \Longleftrightarrow \text { linear operator } \hat{F} \text {. }
$$

To compare predictions of the theory with experimental data it was necessary to understand how one can determine the values of dynamical variables in the given state. W.Heisenberg [3] gave a partial answer to this problem:

If matrix that corresponds to the dynamical variable is diagonal, then its diagonal elements define possible values for the dynamical variable, i.e. its spectrum.

$$
(\hat{F})_{m n}=f_{m} \delta_{m n} \Longleftrightarrow\left\{f_{m}\right\} \quad \text { is spectrum } \mathcal{F} \text {. }
$$

\subsection{Quantum State}

The general solution of the problem was given by von Neumann in 1927. He proposed the following procedure for calculation of average values of physical variables:

$$
<\mathcal{F}>=\operatorname{Tr}(\hat{F} \hat{\rho}) \text {. }
$$

It is possible to represent the operator $\hat{F}$ in the form

$$
\hat{F}=\sum_{n, m}\left|\psi_{n}\right\rangle\left\langle\psi_{n}|\hat{F}| \psi_{m}\right\rangle\left\langle\psi_{m}\right|=\sum_{n, m} F_{n m} \hat{P}_{m n},
$$

where $\left\{\mid \psi_{m}\right\}$ is a basis in Hilbert space and

$$
\hat{P}_{m n}=\left|\psi_{n}\right\rangle\left\langle\psi_{m}\right| .
$$

The average value of the variable $\hat{F}$ is

$$
<\hat{F}>=\sum_{n, m} F_{n m} \rho_{m n},
$$

where

$$
\rho_{m n}=<\hat{P}_{m n}>\text {. }
$$

If we suppose that the numbers $\rho_{m n}$ define the operator $\hat{\rho}:\left\langle\psi_{n}|\hat{\rho}| \psi_{m}\right\rangle=\rho_{m n}$ then the average of the variable $\hat{F}$ is represented in the form (1). 
Really, when they suppose that the operator $\hat{\rho}$ does not depend of the variable $\hat{F}$ but only depends on the physical state of the quantum system they introduce the proposition that the theory is noncontextual. All subsequent experiments confirmed quantum mechanical theory.

Operator $\hat{\rho}$ have to satisfy three conditions:

$$
\begin{gathered}
\text { 1) } \hat{\rho}^{+}=\hat{\rho}, \\
\text { 2) } \operatorname{Tr} \hat{\rho}=1, \\
\text { 3) } \forall \psi \in \mathcal{H} \quad<\psi \mid \hat{\rho} \psi>\geq 0 .
\end{gathered}
$$

By the formula for average values von Neumann found out the correspondence between linear operators $\hat{\rho}$ and states of quantum systems:

$$
\text { state of a system } \rho \Longleftrightarrow \text { linear operator } \hat{\rho} \text {. }
$$

In this way, the formula for average values becomes quantum mechanical definition of the notion "a state of a system". The operator $\hat{\rho}$ is called Density Matrix.

If $\hat{F}$ is an observable with pure discrete spectrum

$$
\hat{F}=\sum_{n} f_{n} \hat{P}_{n}
$$

then

$$
\langle\hat{F}\rangle=\sum_{n} f_{n} \operatorname{Tr}\left(\hat{P}_{n} \hat{\rho}\right) .
$$

Therefore, $\operatorname{Tr}\left(\hat{P}_{n} \hat{\rho}\right)$ is a probability of an observable $\hat{F}$ gets a value $f_{n}$ in the state $\hat{\rho}$.

Since density matrix is a positive definite operator and its trace equals 1 , we see that its spectrum is pure discrete and it can be written in the form

$$
\hat{\rho}=\sum_{n} p_{n} \hat{P}_{n}
$$

where $\hat{P}_{n}$ is a complete set of self-conjugate projective operators:

$$
\hat{P}_{n}^{+}=\hat{P}_{n}, \quad \hat{P}_{m} \hat{P}_{n}=\delta_{m n} \hat{P}_{m}, \quad \sum_{n} \hat{P}_{n}=\hat{E} .
$$

Numbers $\left\{p_{n}\right\}$ satisfy the condition

$$
p_{n}^{*}=p_{n}, \quad 0 \leq p_{n}, \quad \sum_{n} p_{n} \operatorname{Tr} \hat{P}_{n}=1 .
$$

It follows that $\hat{\rho}$ acts according to the formula

$$
\hat{\rho} \Psi=\sum_{n} p_{n} \sum_{\alpha \in \Delta_{n}} \phi_{n \alpha}\left\langle\phi_{n \alpha} \mid \Psi\right\rangle .
$$

The vectors $\phi_{n \alpha}$ form an orthonormal basis in the space $\mathcal{H}$. Sets $\Delta_{n}=\left\{1, \ldots, k_{n}\right\}$ are defined by degeneration multiplicities $k_{n}$ of eigenvalues $p_{n}$. 


\subsection{Dispersion and Pure States}

From the properties of density matrix and the definition of positively definite operators:

$$
\hat{F}^{+}=\hat{F}, \quad \forall \psi \in \mathcal{H} \quad<\psi \mid \hat{F} \psi>\geq 0
$$

it follows that the average value of nonnegative variable is nonnegative. Moreover, the average value of nonnegative variable is equal to zero if and only if this variable equals zero. Now it is easy to give the following definition:

variable $\mathcal{F}$ has a definite value in the state $\rho$ if and only if its dispersion in the state $\rho$ is equal to zero.

The dispersion of a quantum variable $\mathcal{F}$ in the state $\rho$ has the form:

$$
\mathcal{D}_{\rho}(\mathcal{F})=\operatorname{Tr}\left(\hat{Q}^{2} \hat{\rho}\right)
$$

where $\hat{Q}$ is an operator:

$$
\hat{Q}=\hat{F}-<\mathcal{F}>\hat{E} .
$$

If $\mathcal{F}$ is observable $\left(\hat{F}=\hat{F}^{+}\right)$then $Q^{2}$ is a positive definite variable. It follows that the dispersion of $\mathcal{F}$ is nonnegative. This makes clear the above-given definition.

The dispersion of the observable $\mathcal{F}$ in the state $\rho$ is given by the equation

$$
\mathcal{D}_{\rho}(\mathcal{F})=\sum_{n} p_{n} \sum_{\alpha \in \Delta_{n}}\left\|\hat{Q} \phi_{n \alpha}\right\|^{2}
$$

All terms in this sum are nonnegative. Hence, if the dispersion is equal to zero, then

$$
\text { if } \quad p_{n} \neq 0, \text { then } \hat{Q} \phi_{n \alpha}=0 \text {. }
$$

Using the definition of the operator $\hat{Q}$, we obtain

$$
\text { if } \quad p_{n} \neq 0 \text {, then } \hat{F} \phi_{n \alpha}=\phi_{n \alpha}\langle F\rangle \text {. }
$$

In other words, if an observable $\mathcal{F}$ has a definite value in the given state $\rho$, then this value is equal to one of the eigenvalues of the operator $\hat{F}$.

In this case we have

$$
\hat{\rho} \hat{F} \phi_{n \alpha}=\phi_{n \alpha} p_{n}\langle\mathcal{F}\rangle, \quad \hat{F} \hat{\rho} \phi_{n \alpha}=\phi_{n \alpha}\langle\mathcal{F}\rangle p_{n}
$$

that proves the commutativity of operators $\hat{F}$ and $\hat{\rho}$.

It is well known, that if $\hat{A}$ and $\hat{B}$ are commutative self-conjugate operators, then there exists self-conjugate operator $\hat{T}$ with non-degenerate spectrum such that $\hat{A}$ and $\hat{B}$ are functions of $\hat{T}$.

Suppose $\hat{F}$ is an operator with non-degenerate spectrum. Then,

if the observable $\mathcal{F}$ with non-degenerate spectrum has a definite value in the state $\rho$, then it is possible to represent the density matrix of this state as a function of the operator $\hat{F}$.

The operator $\hat{F}$ can be written in the form

$$
\hat{F}=\sum_{n} f_{n} \hat{\Pi}_{n}
$$




$$
\hat{\Pi}_{n}^{+}=\hat{\Pi}_{n}, \quad \hat{\Pi}_{m} \hat{\Pi}_{n}=\delta_{m n} \hat{\Pi}_{m}, \quad \operatorname{tr}\left(\hat{\Pi}_{n}\right)=1, \quad \sum_{n} \hat{\Pi}_{n}=\hat{E} .
$$

The numbers $\left\{f_{n}\right\}$ satisfy the conditions

$$
f_{n}^{*}=f_{n}, \quad f_{n} \neq f_{n^{\prime}}, \quad \text { if } \quad n \neq n^{\prime} .
$$

From

$$
\langle F\rangle=\sum_{n} p_{n} f_{n}=f_{N}, \quad\left\langle F^{2}\right\rangle=\sum_{n} p_{n} f_{n}^{2}=f_{N}^{2}
$$

we get

$$
p_{n}=\delta_{n N}
$$

In this case density matrix is a projective operator satisfying the condition

$$
\hat{\rho}^{2}=\hat{\rho}
$$

It acts as

$$
\hat{\rho} \Psi=\hat{\Pi}_{N}|\Psi\rangle=\Psi_{N}\left\langle\Psi_{N} \mid \Psi\right\rangle
$$

where $\left|\Psi_{N}\right\rangle$ is a vector in Hilbert space. It is so-called pure state.

\subsection{Density Matrix and Gleason Theorem}

To each observable there corresponds a set of orthogonal projection operators $\left\{\Pi_{i}\right\}$ over a complex Hilbert space $\mathcal{H}$ that form a decomposition of the identity

$$
\sum_{n} \hat{\Pi}_{n}=\hat{E}
$$

Quantum mechanics dictates that it is expected the various outcomes with a probability

$$
p_{n}=\operatorname{Tr}\left(\hat{\rho} \hat{\Pi}_{n}\right)
$$

It was assumed by von Neumann and then was finally proven by Gleason[6] in 1957 as the following theorem:

Assume there is a function $f$ from the one-dimensional projectors acting on a Hilbert space of dimension greater than 2 to the unit interval, with the property that for each orthonormal basis $\left\{\left|\psi_{k}\right\rangle\right\}$,

$$
\sum_{k} f\left(\left|\psi_{k}\right\rangle\left\langle\psi_{k}\right) \mid=1\right.
$$

Then there exists a density matrix operator $\hat{\rho}$ such that

$$
f(|\psi\rangle\langle\psi|)=\langle\psi|\hat{\rho}| \psi\rangle .
$$

It assumes that each orthonormal basis corresponds to mutually exclusive results of measurement of some observable. The task is to derive the probabilities for the measurement outcomes. The only requirement is that the probability for obtaining the result corresponding to a normalized vector $|\psi\rangle$ depends only on $|\psi\rangle$ itself, not on the other vectors in the orthonormal basis defining a particular measurement. This important assumption is called the "noncontextuality". It means that the probabilities are consistent with the Hilbert-space structure of observables. With these assumptions the probabilities for all measurements can be derived from a density matrix using the standard quantum probability rule. 


\section{Conditional Density Matrix}

\subsection{Composite System and Reduced Density Matrix}

Suppose that the Hilbert space $\mathcal{H}$ is a direct product of two Hilbert spaces $\mathcal{H}_{1}, \mathcal{H}_{2}$ :

$$
\mathcal{H}=\mathcal{H}_{1} \otimes \mathcal{H}_{2} .
$$

Suppose the composite indexes $m, \quad n, \ldots$ are divided into two parts: $m=\{r, u\} ; n=$ $\{s, v\}, \ldots$ So, there is a basis in the space that can be written in the form

$$
|\phi\rangle_{n}=|f\rangle_{r}|g\rangle_{v}
$$

In quantum mechanics it means that the system $S$ is a unification of two subsystems $S_{1}$ and $S_{2}$ :

$$
S=S_{1} \cup S_{2} \text {. }
$$

The Hilbert space $\mathcal{H}$ corresponds to the system $S$ and the spaces $\mathcal{H}_{1}$ and $\mathcal{H}_{2}$ correspond to the subsystems $S_{1}$ and $S_{2}$.

If quantum state of the composite system is density matrix $\rho_{1+2}$ then the state of the subsystem $S_{1}$ is defined by Reduced Density matrix

$$
\hat{\rho}_{1}=\operatorname{Tr}_{2} \hat{\rho}_{1+2},
$$

the reduced density matrix for the subsystem $S_{2}$ is

$$
\hat{\rho}_{2}=\operatorname{Tr}_{1} \hat{\rho}_{1+2} .
$$

Quantum states $\rho_{1}$ and $\rho_{2}$ of subsystems are defined uniquely by the state $\rho_{1+2}$ of the composite system.

\subsection{Conditional Probabilities}

We recall some definitions of probability theory.

Let $h$ be an event with positive probability. For any event A we define

$$
\mathbf{P}\{A \mid h\}=\frac{\mathbf{P}\{A h\}}{\mathbf{P}\{h\}} .
$$

This is conditional probability of the event A for given event $h$.

This formula can be written in the form:

$$
\mathbf{P}\{A h\}=\mathbf{P}\{A \mid h\} \mathbf{P}\{h\} .
$$

Let $h_{1}, \ldots h_{n}$ be a set of mutually exclusive events such that one of them takes place necessarily. Then any event $A$ can take place only with one of the events $h_{j}$. It can be written as

$$
A=A h_{1} \cup A h_{2} \cup \ldots \cup A h_{n} .
$$

Since $A h_{j}$ are mutually independent their probabilities are added.

Thus,

$$
\mathbf{P}\{A\}=\sum_{j} \mathbf{P}\left\{A \mid h_{j}\right\} \mathbf{P}\left\{h_{j}\right\} .
$$

This is well-known formula for total probability in terms of conditional probabilities. 


\subsection{Conditional Density Matrix}

Let the operators $\hat{P}_{n}^{(2)}$ be the projections on certain basis states in the Hilbert space $\mathcal{H}_{2}$ of pure states of subsystem $S_{2}$ :

$$
\hat{P}_{n}^{(2)}=\left|u_{n}\right\rangle\left\langle u_{n}\right|, \quad \sum_{n} \hat{P}_{n}^{(2)}=\hat{E} .
$$

According definition the reduce density matrix for subsystem $S_{1}$ is

$$
\begin{gathered}
\rho_{s r}^{(1)}=\sum_{u v} \delta_{u v} \rho_{s v ; r u}=\sum_{u v} \sum_{n}\left(\hat{P}_{n}^{(2)}\right)_{u v}(\hat{\rho})_{s v ; r u}= \\
\sum_{n} \sum_{u}\left(\hat{P}_{n}^{(2)} \hat{\rho}\right)_{s u ; r u}=\sum_{n} p_{n} \frac{\sum_{u}\left(\hat{P}_{n}^{(2)} \hat{\rho}\right)_{s u ; r u}}{p_{n}} .
\end{gathered}
$$

Therefore, the reduced density matrix $\rho^{(1)}$ is written in the form:

$$
\hat{\rho}^{(1)}=\sum_{n} p_{n} \hat{\rho}_{n}^{(c)}
$$

where

$$
p_{n}=\sum_{u v} P_{n}^{(2)}(u \mid v) \sum_{r} \rho_{r v ; r u}=\sum_{u v} P_{n}^{(2)}(u \mid v) \hat{\rho}^{(2)}(v, u)
$$

or

$$
p_{n}=\operatorname{Tr}_{2}\left(\hat{P}_{n}^{(2)} \hat{\rho}^{(2)}\right) .
$$

If the set of projections $\hat{P}_{n}^{(2)}$ is associated with some observable in the subsystem $S_{2}$

$$
\hat{G}=\sum_{n} g_{n} \hat{P}_{n}^{(2)}
$$

then $p_{n}$ is a probability of the variable $\hat{G}$ gets a value $g_{n}$ in the state $\hat{\rho}^{(2)}$.

The operator $\hat{\rho}_{n}^{(c)}$ equals:

$$
\rho_{n}^{(c)}(r \mid s)=\frac{1}{p_{n}} \sum_{u v} P_{n}^{(2)}(u \mid v) \rho_{r v ; s u}
$$

and satisfies all conditions (1). It is density matrix or quantum state.

Since $w_{r}=\hat{\rho}_{r r}^{(1)}$ is a probability to find a subsystem $S_{1}$ in the state $|r\rangle$, we see that an equality

$$
w_{r}=\sum_{n} p_{n} \rho_{n}^{(c)}(r \mid r)
$$

is formula $(2)$.

Then the operator $\hat{\rho}_{n}^{(c)}$ is called conditional density matrix and is written [5]

$$
\hat{\rho}_{1 / 2 n}^{(c)}=\frac{\operatorname{Tr}_{2}\left(\hat{P}_{n}^{(2)} \hat{\rho}\right)}{\operatorname{Tr}\left(\hat{P}_{n}^{(2)} \hat{\rho}\right)}=\frac{\operatorname{Tr}_{2}\left(\hat{P}_{n}^{(2)} \hat{\rho}\right)}{w_{n}} .
$$

This is a conditional density matrix, i.e. a quantum state, for subsystem $S_{1}$ under condition that the subsystem $S_{2}$ is selected in pure state $\hat{P}_{n}^{(2)}$. It is the most interesting case 
for quantum communication. This definition of the quantum state of quantum subsystem assumes noncontextual approach in quantum mechanics.

It is necessary to note that although formula (4) arose in description of measurement, for example in papers [11], [8] and et. , it was presented as the result of transformation of quantum state of the system during measurement. Here, conditional density matrix is the definition of a new quantum state of the subsystem that is selected under definite physical condition.

\subsection{Conditional Density Matrix in Case of Generalized Measure- ment}

It was recently shown [9] that a Glison-like theorem can be easy proved (and also extends to the case of 2-dimensional Hilbert space) on a set of effects $\{E\}$. It is a set of projections but commutativity (or orthogonality) is no longer necessary. According to this theorem

any generalized probability measure is of the form $E \rightarrow v(E)=\operatorname{tr}[\rho E]$ for all $E$, for some density operator $\rho$.

While we consider a generalized measurement [8] in the subsystem $S_{2}$ we suppose that a set of projections $\left\{\hat{E}_{b}\right\}$ exits and satisfies the properties

$$
<\psi\left|\hat{E}_{b}\right| \psi>\geq 0, \quad \forall \mid \psi>, \quad \sum_{b} \hat{E}_{b}=\hat{I}^{(2)} .
$$

The probabilities of outcomes are equal

$$
P(b)=\operatorname{tr}\left(\hat{\rho} \hat{E}_{b}\right) .
$$

We don't suppose that condition $\Pi_{i} \Pi_{j}=\delta_{i j} \Pi_{i}$ is fulfilled.

In this case the decomposition (3) is also valid and quantum state of subsystem $S_{1}$ under condition that the subsystem $S_{2}$ is selected in pure state $\hat{E}_{b}$ is

$$
\hat{\rho}_{1 / 2 b}^{(c)}=\frac{\operatorname{Tr}_{2}\left(\hat{E}_{b} \hat{\rho}\right)}{\operatorname{Tr}\left(\hat{E}_{b} \hat{\rho}\right)} .
$$

\section{Conditional Density Matrix Description of Entan- glement Swapping}

In the experiments [2] with installation two pairs of correlated photons are emerged simultaneously. The polarization state of the system is being described by the simultaneous wave function

$$
\left|\Psi\left(\sigma_{1}, \sigma_{2}, \sigma_{3}, \sigma_{4}\right)\right\rangle=\Psi_{-}\left(\sigma_{1}, \sigma_{2}\right) \Psi_{-}\left(\sigma_{3}, \sigma_{4}\right),
$$

where $\Psi_{-}$is antisymmetric state of pair of photons

$$
\Psi_{-}\left(\sigma_{i}, \sigma_{j}\right)==\frac{1}{\sqrt{2}}\left(\chi_{0}\left(\sigma_{i}\right) \chi_{1}\left(\sigma_{j}\right)-\chi_{1}\left(\sigma_{i}\right) \chi_{0}\left(\sigma_{j}\right)\right)
$$


and $\chi_{s}(\sigma)$ are two basis states with orthonormal polarization. Reduced density matrix of subsystem $S_{1-4}$ is proportional to unity

$$
\rho_{14}=\frac{1}{2} \hat{I}^{(1)} \otimes \frac{1}{2} \hat{I}^{(4)}
$$

But if we select the pair of photons 1-4 only under condition that pair 2-3 is in the pure state $\Psi_{-}\left(\sigma_{2}, \sigma_{3}\right)$ then quantum state of pair 1-4 is conditional density matrix

$$
\hat{\rho}_{14 / 23}^{c}=\frac{\operatorname{Tr}_{23}\left(\hat{P}_{23} \hat{\rho}_{1234}\right)}{\operatorname{Tr}\left(\hat{P}_{23} \hat{\rho}_{1234}\right)},
$$

where operator $\hat{P}_{23}$ selects pair $2-3$ is pure state $\Psi_{-}\left(\sigma_{2}, \sigma_{3}\right)$. Direct calculation shows that the pair of the photons ( 1 and 4 ) has to be in pure state with the wave function $\Phi\left(\sigma_{1}, \sigma_{4}\right)=$ $\Psi_{-}\left(\sigma_{1}, \sigma_{4}\right)$. As the system $S_{1234}$ is described by simultaneous wave function the time order of measurements has no importance.

Other examples demonstrating the utilization of conditional density matrix in different schemes of quantum communication are represented in [10].

\section{Conclusion}

Provided that the subsystem $S_{2}$ of composite quantum system $S=S_{1}+S_{2}$ is selected in a pure state $\hat{P}_{n}$ the quantum state of subsystem $S_{1}$ is conditional density matrix $\hat{\rho}_{1 c / 2 n}$. Reduced density matrix $\hat{\rho}_{1}$ is connected with conditional density matrices by an expansion $(3)$.

\section{References}

[1] J.von Neumann, Gött. Nach. pp. 1-57, 245 - 272, 1927. See, also, J. von Neumann, "Mathematische Grundlagen der Quantenmechanik", (Berlin) 1, 1932.

[2] T. Jennewein, G. Weihs, J.-W. Pan and A. Zeilinger, Phys.Rev. Lett. 88, 017903 (2002).

[3] W.Heisenberg, Zeits.f.Physik 43 , 172, (1925).

[4] V.V.Belokurov, O.A.Khrustalev, O.D.Timofeevskaya, "Quantum Teleportation: an ordinary miracle", (Izgevsk, Regular and Haotic Dynamics) 1, 2000.

[5] V.V.Belokurov, O.A.Khrustalev, V.A.Sadovnichy, O.D.Timofeevskaya in System and subsystems in quantum communication, (Proceedings of XXII Solvay Conference on Physics, Delphi Latin, 2001), World Scientific Publising, 555, 2003.

[6] A.M.Gleason, J.Math.Mech. 6, 885 (1957).

[7] H.Barnum, C.M.Caves, J.Finkelstein, C.A.Fuchs and R.Schack, arXive: quantph/9907024 (1999). 
[8] C.A.Fuchs, arXive: quant-ph/0106166 (2001).

[9] P.Busch, arXive: quant-ph/9909073 (2003).

[10] V.V.Belokurov, O.A.Khrustalev, V.A.Sadovnichy, O.D.Timofeevskaya, Partucles and Nuclei, Lett. 1, 116 (2003).

[11] A.Barchielly, Phys.Rev D 32, 347 (1985). 